\title{
Solving Singular Control Problems using Uniform Trigonometrization Method
}

\author{
Kshitij Mall ${ }^{1}$, Ehsan Taheri ${ }^{1}$, and Prathamesh Prabhu ${ }^{2}$ \\ ${ }^{1}$ Auburn University \\ ${ }^{2}$ Purdue University
}

May 29, 2020

\begin{abstract}
This study investigates the application of a recently developed construct, the Uniform Trigonometrization Method (UTM), to the singular control problems in chemical engineering. The UTM involves minimal modifications to the original problem, thereby generating near-singular control solutions that can be used for conceptual design and serve as an alternate to direct techniques like nested and simultaneous approaches. Eight classical singular control problems with known analytical solutions and three complex singular control problems from chemical engineering domain are solved in this study. The results obtained using the UTM for these problems are found to match well with the literature and are of higher resolution as compared to the results obtained using a direct pseudospectral based solver. The ability of the UTM to handle complex chemical engineering problems with both singular controls and state path constraints has also been demonstrated in this study.
\end{abstract}

\section{Hosted file}

UTM_AIChE_KMall.pdf available at https://authorea.com/users/327869/articles/455265-solvingsingular-control-problems-using-uniform-trigonometrization-method 